\title{
Mesenteric Lipoma with Volvulus: A Rare Cause of Acute Abdomen in a Child
}

\author{
Hamza Malik, Bilal Mirza, Nabila Talat, Waseem Zia and Anum Manzoor \\ Department of Pediatric Surgery, The Children's Hospital and the Institute of Child Health, Lahore, Pakistan
}

\begin{abstract}
Lipoma is benign tumor of mature fat cells and is reported to develop anywhere in the body, e.g. trunk and extremities; however, lipoma in the mesentery of gut is rare, particularly in children. Mesenteric lipomas may be asymptomatic or may present with acute abdomen by leading to volvulus of gut. We report a 6-year female with intestinal obstruction. Exploratory laparotomy revealed a large mesenteric lipoma in the mesentery of small bowel causing volvulus of ileum. Lipoma was excised and postoperative recovery remained uneventful both in the hospital and on subsequent follow up. We believe mesenteric lipoma should be considered as a cause of volvulus and in differential diagnosis of acute abdomen in children.
\end{abstract}

Key Words: Mesentery, Lipoma, Volvulus, lleum, Intestinal obstruction.

How to cite this article: Malik H, Mirza B, Talat N, Zia W, Manzoor A. Mesenteric Lipoma with Volvulus: A Rare Cause of Acute Abdomen in a Child. J Coll Physicians Surg Pak 2020; 30(06):650-651 https://doi.org/10.29271/jcpsp.2020.06.650.

\section{INTRODUCTION}

Lipoma is a common tumor of mature fat cells in adults and children. It may be single or multiple, and may occur anywhere in the body. However, lipoma in mesentery of gut in children is rare. ${ }^{1}$ Most previously reported cases presented as painless abdominal masses. ${ }^{2}$ We report a case of mesenteric lipoma presenting as acute abdomen in a 6-year girl. On perusal of literature, less than 50 pediatric cases of mesenteric lipoma have been reported, mainlyascase reports. ${ }^{3}$

\section{CASE REPORT}

A 6-year girl presented with 24 hours history of acute abdominal pain, vomiting and constipation. There was past history of mild abdominal discomfort, relieved by medications. On examination, patient was irritable, dehydrated; and had abdominal distension, and generalised tenderness. No definitive mass could be appreciated on clinical examination. Abdominal radiographs in the erect posture showed multiple air fluid levels. After resuscitation, she had an exploratory laparotomy. There was $10 \times 8 \mathrm{~cm}$ fatty mass in the mesentery of distal ileum causing volvulus of gut (Figure $1 \mathrm{~A})$. Another small $(2 \times 2 \mathrm{~cm})$ fatty mass was present in the mesentery. After de-twisting of volvulus and ensuring the viability of gut, both fatty masses arising from mesentery were enucleated (Figure 1B, C). Histopathology revealed lipoma and there was no evidence of malignancy (Figure 1D).

Correspondence to: Prof. Nabila Talat, Department of Pediatric Surgery Unit II, The Children's Hospital and the Institute of Child Health, Lahore, Pakistan

E-mail: nabila.talat@chich.edu.pk

Received: May 09, 2019; Revised: September 15, 2019;

Accepted: September 17, 2019

DOI: https://doi.org/10.29271/jcpsp.2020.06.650
Postoperative recovery was uneventful and there was no recurrence at 6 months follow-up as assessed by ultrasonography.
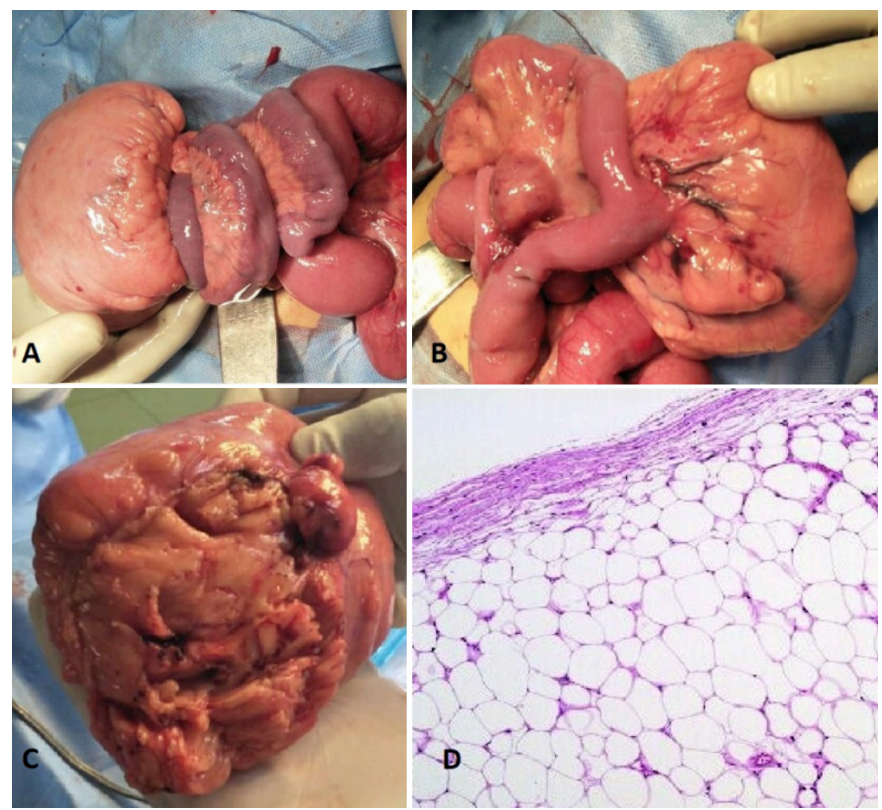

Figure 1: (A) Mesenteric lipoma with volvulus of the distal ileum. The color change of the distal ileum is quite obvious. (B) Mesenteric lipoma after de-torsion. (C) Mesenteric lipoma excised from the mesentery of distal ileum. (D) Histopathology of specimen showing an encapsulated tumor composed of mature fatcells.

\section{DISCUSSION}

Lipoma is a benign, well encapsulated and slow growing tumor of mature fat cells occurring predominantly in adults. These can arise in any part of the body but mesenteric lipomas are rare. ${ }^{1,4}$ Mesenteric lipomas usually present as asymptomatic abdominal masses or may cause nonspecific symptoms, e.g., abdominal distension, anorexia, and constipation. ${ }^{2}$ Rarely mesenteric 
lipomas can cause intestinal obstruction due to torsion or volvulus of involved segment of gut as found in the present case. $^{3,5}$

Diagnosis of mesenteric lipomas is rarely made preoperatively. Ultrasound abdomen, MRI or CT scan abdomen are useful in the diagnosis; and also to differentiate these from other mesenteric masses. In case of lipoma causing volvulus, the doppler ultrasound and CT scan can pick a fat density mass with whirlpool or swirl sign. ${ }^{3,5}$

Treatment of mesenteric lipoma depends upon size and site of lipoma and presence ofcomplications. Simpleexcision/enucleation of lipoma from leaves of mesentery is performed in case the bowel is not compromised. ${ }^{1,4}$ Excision of lipoma with resection of involved segment of gut and end-to-end anastomosis has to be performed in case of gangrenous bowel or if lipoma is too proximate and compressing the small bowel. ${ }^{3-5}$ In the present case, enucleation of lipoma was done after de-torsion of volvulus as gut was not compromised. In addition, another small lipoma in the adjacent mesentery was also removed, fearing its further growth can cause complications.

Mesenteric lipomas can recur after excision in $5 \%$ of cases due to incomplete excision. ${ }^{2}$ Therefore, a long term follow-up with ultrasound should be done as lipomas are slow growing lesions. CT scan should be reserved in case a suspicion arises on ultrasonography or a strong clinical suspicion of recurrence arises. The patient was followed up with ultrasonography and she is doing fine till date.

In conclusion, mesenteric lipoma, thoughrare, should beconsidered in the differential diagnoses of acute intestinal obstruction in young children with or without a palpable abdominal mass. Preoperative diagnosis is rare in acute presentation; however,
CTscancan help diagnosing italong with its complications.

\section{PATIENT'S CONSENT:}

Informed consent was taken from the patient's parents for publication of this case report and related images.

\section{CONFLICT OF INTEREST:}

No conflict of interest is declared.

\section{AUTHORS' CONTRIBUTION:}

HM: Data acquisition, literature review, manuscript drafting.

BM: Operating surgeon, manuscript review, editing, critical review.

NT: Supervision, critical revision, final approval.

WZ: Operating surgeon, critical revision.

AM: Assistance in manuscript designing and literature review.

\section{REFERENCES}

1. Kaniklides C, Frykberg T, Lundkvist K. Paediatric mesenteric lipoma, an unusual cause of repeated abdominal pain. Acta Radiol 1998; 39(6):695-7.

2. Tayeh C, Mneimneh S, El-Masri R, Daoud N, Rajab M. Giant mesenteric lipoma: A case report and a review of the literature. J Pediatr Surg Case Reports 2019; 3(4): 166-70.

3. Alsayegh RO, Almutairi R, Taqi E, Alnaqi A. Mesenteric lipoma presenting as small bowel volvulus. J Pediatr Surg Case Reports 2019; 43:47-9.

4. Enyinnah M, Umezurike C. Mesenteric lipoma causing recurrent intestinal obstruction. Niger J Clin Pract 2013; 16(4):551-3.

5. El Mouhadi S, Chat L, Dafiri R. Giant mesenteric lipoma: An uncommon cause of volvulus in a child. Eurorad 2010; 2010:8146. 